\title{
RESIDUAL GAS EFFECT ON PHOTOFIELD EMISSION FROM TUNGSTEN (111) FACE
}

\author{
P. Hądzel, T. Radoń and S. Jaskólka \\ Institute of Experimental Physics, University of Wroclaw \\ Pl. Maxa Borna 9, 50-204 Wrocław, Poland
}

(Received February 19, 1997; in final form April 15, 1997)

\begin{abstract}
Using the photofield emission method, surface states and bulk excitations from clean and residual gas adsorbed (111) face of tungsten were measured. Residual gas shaded the surface states and diminished photoemission from the bulk excitations. Gas of the artificially increased background pressure $10^{-8} \mathrm{~Pa}$ adsorbed during the measurement deforms the photocurrent-voltage characteristic; the optical excitations observed for clean surfaces are exhibited, diminished or lost. For the adsorbed (111) face the state related to the metal-gas surface has been observed.

PACS numbers: 79.70.+q, 79.60.Dp, 73.20.Hb
\end{abstract}

\section{Introduction}

A study of the electronic states below the Fermi level and above the vacuum level is accessible by photoemission. The electronic states lying between the Fermi level and the vacuum level can be detected by the inverse photoelectric effect [1] and by the photofield emission. In the photofield emission, an electron is excited from an occupied electronic state below the Fermi level to an unoccupied state below the vacuum level and then emitted to the vacuum. In the presence of a strong electric field applied to a field emitter the Shottky lowering of the surface potential barrier exists. Then the photoexcited electron can tunnel through the barrier or can be emitted above the barrier to the vacuum and the final energy of the emitted photoelectron is connected with the band structure between the Fermi level and the vacuum level.

First experimental observations of the effect of the band structure on photofield emission current-voltage characteristics were made many years ago [2-4]. It has been shown later that the photocurrent curves can give some detailed information about the electronic states in the vicinity of the Fermi level [5]. Using linearly polarized light and an appropriate angle of light incidence, the dominant surface excitations were observed when the perpendicular component of the electric vector of the light beam was prevailing [6]. When the electric vector of the light was parallel to the emitting surface, the photocurrent due to the bulk photoexcitations was observed $[7,8]$. 
The photofield emission current-voltage characteristics show various slopes and shoulders, and the shoulders are considered as correlated with optical transitions between the energy bands of the emitter $[9,10]$. The final and initial energies are evaluated from the excitation energy and the measured lowering of the barrier maximum. The energies of excited electrons have a discrete character which is connected with the excitation rules. Therefore, the direct transitions and also the indirect ones in the band structure commonly give several groups of photoelectrons having different final energies. Then the groups induce the increased photocurrent which is observed in a photocurrent-voltage characteristic as a few shoulders concerning the different groups of photoelectrons. From the voltages at which the shoulders occur, and the geometrical factor estimated by Fowler-Nordheim plots, the field strength at the emitter surface and then the lowering of the top of the surface barrier are obtained. According to the interpretation [11] and good experimental confirmation $[9,5]$, the shoulders occur when the top of the barrier is lowered towards the energy of an excited electron group, then the barrier maximum indicates the final energy of the excited electrons. The difference between the final and photon energy gives the initial energy of electrons. It can be assumed for small photon energies (a few eV) that the photoelectrons do not lose any energy. In a rough approximation, as long as the final energy of photoelectrons is lower than the barrier maximum, the photoelectrons pass through the barrier. When the final energy of photoelectrons exceeds the barrier, we observe a shoulder in the photocurrent-voltage characteristic. In this case, the photoelectrons emitted above the barrier compose the main part of the photocurrent and the photocurrent remains constant until the photoelectrons from the next group with a lower energy start to pass through the barrier and, at a higher voltage, above the barrier.

In this paper we present: (a) a considerable effect of a small portion of residual gas on surface states and the emission from bulk excitations, (b) the effect of gas on the shape of photocurrent curves measured from a clean emitter at a higher pressure, i.e. at $10^{-8} \mathrm{~Pa},(\mathrm{c})$ the appearance of the surface state related to the gas affected metal surface.

\section{Experimental}

The photofield emission currents are in the range of $10^{-13}-10^{-11} \mathrm{~A}$. Such very small currents are difficult to measure, they are disturbed mainly by flicker noise connected with the residual gas adsorption and diffusion on the field emitter surface. Therefore, the photofield emission measurements must be made in ultra-high vacuum, i.e. at the residual gas pressure in a range of $10^{-10}-10^{-9} \mathrm{~Pa}$. The vacuum needed might be provided in this experiment owing to the small volume (about 1 liter) of the field emission tube sealed off from the pumping system and equipped with titanium and molybdenum getters specially prepared. The background gas pressure in the ultra-high vacuum was varied by heating a tungsten wire which was present in the field emission microscope. Upon typical heating of the wire, the pressure reached a value of $p=3 \times 10^{-7} \mathrm{~Pa}$ and after 2-3 seconds decreased to $p=10^{-8} \mathrm{~Pa}$. Data on the pressure of residual gases are of importance in measurements, which will be discussed later. 
In order to measure very small photocurrents, phase-sensitive detection was applied [3]. The constant field emission current was separated from the alternating photofield emission current caused by a modulation of the light beam. The laser light beam $(h \nu=1.96 \mathrm{eV})$ was chopped with a frequency of $8500 \mathrm{~Hz}$ and the alternating photocurrent was amplified. Such a high frequency was used to decrease the thermocurrent caused by the heating of the field emitter. The irradiance at the emitter tip was smaller than $20 \mathrm{~W} / \mathrm{cm}^{2}$ and for some measurements was specially diminished to avoid the thermocurrents. The angle of incidence of the light beam onto the plane of the tungsten microcrystal was usually about $70^{\circ}$. This ensured a dominant component of the electric vector of the polarized light to have either parallel ( $s$-polarized light) or perpendicular ( $p$-polarized light) orientation to the emitting surface so as to obtain the photocurrent mainly either from the volume or from the surface of the emitter, respectively.

The lowering of the surface barrier was estimated from the geometrical factor $\beta$ of the emitter tip and the applied voltage. The factor $\beta$ was obtained from the Fowler-Nordheim characteristic assuming the average work function to be $4.52 \mathrm{eV}$ for clean tungsten. The work function of the (111) face of tungsten crystal was assumed to be $4.40 \mathrm{eV}$ [12], and the work function after the gaseous adsorption was estimated from the photoemission threshold. In this case the work function is the sum of the photon energy and the magnitude of the lowering of the surface barrier.

The measurements were controlled by using a computer program. The duration of measurement of each value of the photocurrent was about $8 \mathrm{~s}$. The time of the measurement of a characteristic was about $400 \mathrm{~s}$. The data is relevant to the influence of residual gas adsorption during the measurements on the shape of photocurrent curves.

\section{Results and discussion}

The results presented here show that the residual gas adsorbed on the emitter destroys the emission effects dependent on optical excitations. Curves 1 and 2 in Fig. 1 taken for different polarization show characteristics obtained for the clean (111) face, and curves 3 and 4 for the surface after the adsorption of gas. The first pair shows distinct shoulders and the second pair gets smooth. It is surprising that a small amount of gas estimated as about 0.2 monolayer adsorbed on the surface caused the disappearance of the shoulders related to the surface states $p_{1}, p_{2}, p_{3}$ and the diminution of emission from the bulk excitations $E_{F}$ and $E_{\mathrm{m}}$ obtained for a clean surface. The surface states and bulk excitations observed earlier [13] are marked in the corner of Fig. 1, $E_{\mathrm{F}}$ as the excitation from the Fermi level and $E_{\mathrm{m}}$ as the excitation to the minimum of the level lying above the Fermi level. A part of the band structure in the $\langle 111\rangle$ direction and the surface density of states of the (111) face of tungsten are based on Christensen and Feuerbacher's calculations [14]. An estimation of the thickness of a small layer of the gaseous adsorbate is as follows. The increase in the work function of the (111) face estimated from the photoemission threshold (the displacement of emission from $E_{\mathrm{F}}$ in curves 1 and 3 ) is only $0.10 \mathrm{eV}$. A field emission pattern of the tungsten single crystal covered by the small portion of residual gas shows changes 


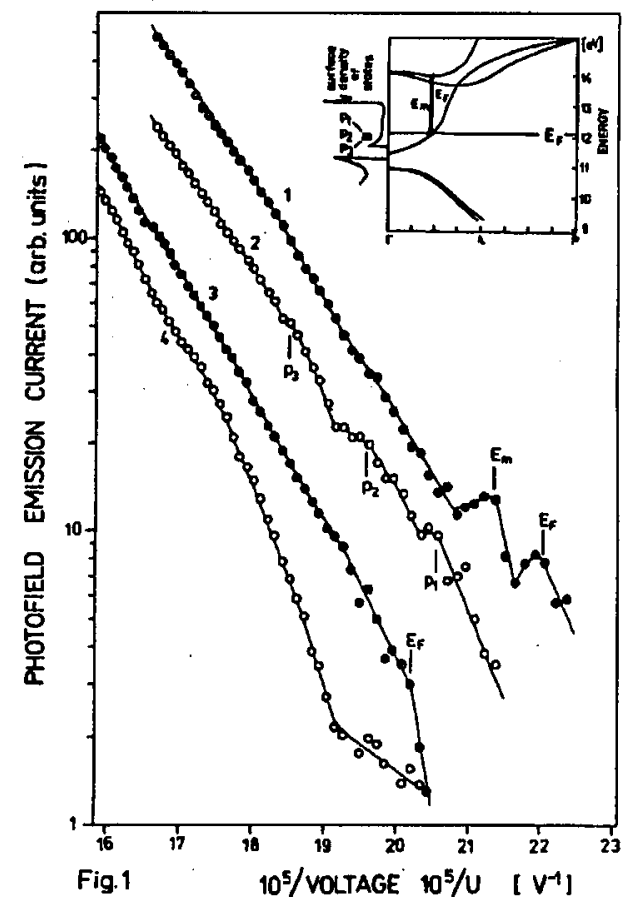



Fig. 1. Photofield emission characteristics from the clean and residual gas adsorbed region (111) of tungsten, taken using photon energy $h \nu=1.96 \mathrm{eV}$. Curves 1 and 2 were obtained for a clean surface, curves 3 and 4 were after gas adsorption. Curves 1 and 3 represent photoemission from the bulk ( $s$-polarized light), curves 2 and 4 represent photoemission from the surface ( $p$-polarized light). In the corner of the figure, a part of the band structure of $\langle 111\rangle$ direction and the surface density of states of the clean (111) face are shown. The excitations in the bulk, $E_{\mathrm{F}}$ and $E_{\mathrm{m}}$, and the excitations from the surface $p_{1}, p_{2}, p_{3}$ are marked.

Fig. 2. Photofield emission characteristics from the $\langle 111\rangle$ direction of tungsten (s-polarized light) using photon energy $h \nu=1.96 \mathrm{eV}$. Curve $1-$ photocurrent from the clean emitter, curves 2 and 3 - photocurrent measured for a higher residual gas pressure (smaller than $10^{-8} \mathrm{~Pa}$ ). The range of voltage and intensity of light beam were smaller than those for measurements shown in Fig. 1.

mainly in the vicinity of the [112] faces. A monolayer of hydrogen increases the work function of the tungsten field emitter by about $0.35 \mathrm{eV}$ [15], while that of oxygen by more than $1.00 \mathrm{eV}[16,17]$, and nitrogen practically does not change the work function [18]. The photocurrent characteristics were measured at room temperature. The heating of the adsorbed field emitter at a temperature of about $600 \mathrm{~K}$ did not change the work function. At this temperature hydrogen should be desorbed [15], but nitrogen and oxygen should stay. The increase in the work function may be ascribed mainly to oxygen adsorption. Assuming that the increase is proportional to the degree of coverage as long as one monolayer is not reached, the oxygen coverage may be estimated as about 0.1 monolayer. A small nitrogen 
coverage is expected because of the very small sticking coefficient for nitrogen $(0.005)$ at $300 \mathrm{~K}$ [19] and the degree of nitrogen coverage seems to be comparable with the coverage of oxygen. The above-mentioned estimations allow us to suppose that the amount of gases adsorbed on the tungsten field emitter, curves 3 and 4 in Fig. 1, was about 0.2 monolayer. Thus the measurements show that the small portion of gas suppressed surface states $\left(p_{1}, p_{2}, p_{3}\right)$ and diminished the emission of electrons excited in the bulk $\left(E_{\mathrm{F}}, E_{\mathrm{m}}\right)$.

Apart from the disappearance of the surface states and the diminution of photoelectrons from the bulk another effect was observed. The influence of residual gas adsorbed on the cleaned emitter surface during the measurement of photocurrent characteristics can be seen in Fig. 2. The curves concern the excitations $E_{\mathrm{F}}$ and $E_{\mathrm{m}}$ in the bulk and they were measured in a range of voltages smaller than that in Fig. 1. Curve 1 represents the measurement on the clean surface, i.e. for the best vacuum $\left(10^{-9} \mathrm{~Pa}\right)$. Then gas was produced by heating the tungsten wire and the pressure increased to about $3 \times 10^{-7} \mathrm{~Pa}$ for 1 second and decreased in about 2 seconds to the level of $10^{-8} \mathrm{~Pa}$. The measurement of curve 3 was begun after cleaning the emitter. The first point in the curve was measured in about 60 seconds after cleaning the emitter, therefore the shoulder $E_{\mathrm{F}}$ is shifted by about $0.02 \mathrm{eV}$ in relation to the shoulder $E_{\mathrm{F}}$ of curve 1 . An important effect of the adsorbed gas during the measurement was a considerable decrease in the photocurrent after reaching the maximum at the shoulder $E_{\mathrm{F}}$, then the current violently increased to reach the shoulder $E_{\mathrm{m}}$ and after that a decrease in the photocurrent was not observed. Curve 2 was measured as the next curve without redosing of residual gas, with continuously pumping getters and after the cleaning of the emitter. The decrease in photocurrent after reaching the maximum at the shoulder $E_{\mathrm{F}}$ is smaller than that in curve 3 , but the current decrease for the shoulder $E_{\mathrm{m}}$ is observed, too. The interpretation of the observations seems to be easy. The condensed gas during the course of measurement decreases the photocurrent continuously by increasing the work function and diminishing the emission. The effect is more visible in the parts of curves where the current is expected to be almost constant, i.e. for voltages higher than those for the excitations $E_{\mathrm{F}}$ and $E_{\mathrm{m}}$ to occur. Since the pressure of the gas was higher during the measurement of curve 3 than that for curve 2 , then the diminishing cffect for the excitation $E_{\mathrm{F}}$ is higher in curve 3 than in curve 2 . For the same reason the segment at the $E_{\mathrm{m}}$ in curve 3 is more smooth and in curve 2 is declined.

Similar effects are observed in characteristics containing shoulders related to the surface states ( $p$-polarized light), which is shown in Fig. 3. Curve 1 was measured in clean vacuum and reveals two surface states $p_{1}$ and $p_{2}$. When the pressure of residual gas in the tube was increased, then the diminishing of current was observed near the shoulder $p_{1}$ and the current of $p_{2}$ smoothed, as can be seen in curve 3 . For a smaller pressure, curve 2 , the shoulder $p_{2}$ is more visible. Moreover, in curves 2 and 3 an unexpected shoulder (marked $P$ ) has appeared, which is situated between the shoulders $p_{1}$ and $p_{2}$. The points near the shoulders $P$ were measured when the surface of the emitter was partially adsorbed. The adsorption rate during the measurement was greater for curve 3 than that for curve 2 and point $P$ is more distinguishable in curve 3 than in curve 2. 

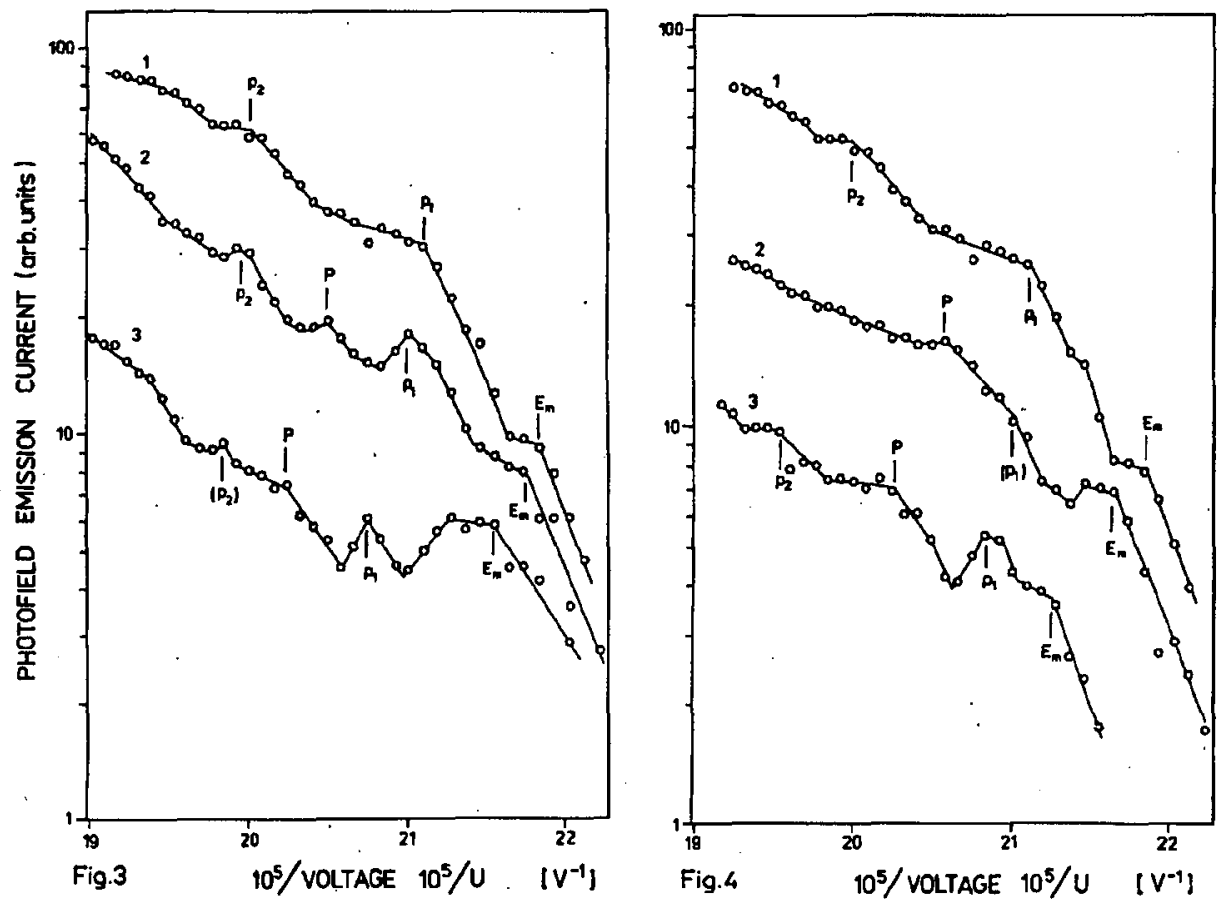

Fig. 3. Photofield emission characteristics from the (111) face of tungsten ( $p$-polarized light) using photon energy $h \nu=1.96 \mathrm{eV}$. Curve 1 - photocurrent from the clean surface, curves 2 and 3 - photocurrent measured for a higher residual gas pressure in the tube (smaller than $10^{-8} \mathrm{~Pa}$ ). The range of voltage and intensity of light beam were smaller than those for measurements shown in Fig. 1.

Fig. 4. Photofield emission characteristics from the (111) face of tungsten ( $p$-polarized light) using photon energy $h \nu=1.96 \mathrm{eV}$. Curve 1 - photocurrent from the clean surface, curve 2 - photocurrent from the gas adsorbed surface after heating at a temperature of about $700 \mathrm{~K}$, curve 3 - after heating at about $900 \mathrm{~K}$. Here the small dark spot on the (111) face was observed.

The state $P$ was observed at the lowest pressure once more for the adsorbed surface, as can be seen in Fig. 4. Curve 1 was measured for a clean emitter surface and curve 3 for the one adsorbed and treated by heating to a temperature of about $700 \mathrm{~K}$. Curve 2 was measured after additional heating of the emitter at a temperature of about $900 \mathrm{~K}$. This heating resulted in the appearance of a small dark spot in the field emission pattern on the region corresponding to the (111) face of the microcrystal of tungsten. For the clean surface of tungsten, the (111) face is indistinguishable on the bright emission region between the $\{112\}$ planes of an emission pattern. The appearance of the state $P$ in Fig. 3 , which is more distinguishable in Fig. 4 (without states $p_{1}$ and $p_{2}$ ) suggests that the state $P$ should be related to the new gas affected metal surface. It is noticeable that the energy of the state $P(-0.12 \mathrm{eV})$ lies between the energies of the states $p_{1}$ and $p_{2}$, i.e. between $-0.08 \mathrm{eV}$ and $-0.15 \mathrm{eV}$ in relation to the Fermi level $E_{\mathrm{F}}$. 
In conclusion, the influence of the residual gas on electron emission is easily noticed in the field emission and particularly in the photofield emission. Adsorption, suriace diffusion and desorption of residual gas molecules occur on the emitter surface and induce photocurrent fluctuations. Therefore the residual gas pressure during photofield emission measurements should be $10^{-9} \mathrm{~Pa}$ or smaller, then the measurements might be reproducible. Moreover, adsorbed molecules "cover" the surface states of the emitter microcrystal, diminish the emission of excited electrons or create their own states. In this case the measurements cannot reflect the properties of a clean surface. The information should be useful to explain some microelectronic problems.

\section{Acknowledgments}

The authors are grateful to Dr S. Surma for his remarks on the manuscript and linguistic corrections. This work was supported by the University of Wrocław within the grant No. 2016/W/IFD/96.

\section{References}

[1] I.K. Lang, Y. Baer, Rev. Sci. Instrum. 50, 221 (1979).

[2] Ch. Kleint, H. Koscheva, in: Abstract 16th Field Emission Symp., Pittsburgh (PA) 1969 , p. 84.

[3] H. Neumann, Ch. Kleint, Ann. Phys. (Leipzig) 27, 237 (1971).

[4] J. Wysocki, Ch. Kleint, Acta Phys. Pol. A 48, 157 (1975).

[5] T. Radoń, Surf. Sci. 100, 353 (1980).

[6] T. Radoń, S. Jaskółka, Surf. Sci. 247, 106 (1991).

[7] T. Radoń, S. Jaskółka, Surf. Sci. 231, 160 (1990).

[8] T. Radoń, S. Jaskółka, E.V. Chulkov, O.S. Koroleva, Vacuum 46, 449 (1995).

[9] T. Radon', Ch. Kleint, Surf. Sci. 60, 540 (1976).

[10] Ch. Kleint, A. Modinos, Surf. Sci. 177, 389 (1986).

[11] Ch. Kleint, in: Intern. Symp. ESCA, Reinhardsbrunn, Extended Abstracts, Karl-Marx-Universität Leipzig, Sektion Chemie, Leipzig 1975, p. 7.

[12] W.S. Fomenko, I.A. Podchernyaeva, Emission and Adsorption Properties of Substances and Materials, Atomizdat, Moscov 1975 (in Russian).

[13] P. Haqdzel, T. Radoń, Vacuum, to be published.

[14] N.E. Christensen, B. Feuerbacher, Phys. Rev. B 10, 2349 (1974).

[15] R. Gomer, R. Westman, R. Lundy, J. Chem. Phys. 26, 1147 (1957).

[16] J. Czyżewski, C. Workowski, Acta Phys. Pol. 33, 913 (1968).

[17] C. Workowski, J. Czyżewski, Acta Phys. Pol. 36, 1095 (1969).

[18] R.S. Polizzotti, G. Ehrlich, J. vhem. Phys. 71, 259 (1979).

[19] P.W. Tamm, L.D. Schmidt, Surf. Sci. 26, 286 (1971). 